\title{
Analysis on Kinematics and Dynamics for a Planar Flexible Driving Target Motion Simulation System
}

\author{
Jianyu $\mathrm{Ma}^{1,2, a}$, Fang Yang ${ }^{2, \mathrm{~b} *}$ and Yujun Xue ${ }^{1,2, \mathrm{c}}$ \\ ${ }^{1}$ Henan University of Science and Technology, Luoyang 471003, China \\ ${ }^{2}$ Henan Key Laboratory of Modern Mechanical Design and Transmission System, Luoyang 471003, \\ China \\ amajianyuvip@163.com, byangfanghkd@163.com, ‘xue_yujun@163.com \\ *The Corresponding author
}

Keywords: Flexible drive motion simulation system; Kinematics analysis; Dynamics analysis; Numerical simulation

\begin{abstract}
The flexible drive have made tremendous inroads into many industrial fields. This paper focuses on the kinematics and dynamics analysis of a planar flexible drive target motion simulation system. The kinematics and dynamics mathematical models of the planar flexible driving target motion simulation system are established. On the basis of this, the simulation analysis of the trajectory of the circle was carried out. The motion law and stress condition of flexible cable under given trajectory are obtained by simulation. The correctness of the model is proved theoretically, and it lays the foundation for further research on precise control.
\end{abstract}

\section{Introduction}

Recently, the flexible drive parallel mechanism has made great progress in many industrial fields[1]. For example, researchers from around the world have developed the product processing, port handling, sea fishing, large diameter Radio Telescope Tracking source motion, aircraft model wind tunnel test of cable driven parallel mechanism prototype[2]. The flexible driving mechanism uses flexible cable instead of the traditional rigid connecting rod[3]. Compared with the traditional rigid drive mechanism, the flexible drive mechanism is simple in structure and high in control accuracy, and different configurations and degrees of freedom can be constructed according to the requirements[4]. Among all these benefits, keeping flexible tension is one of the fundamental challenges of flexible transmission. On the other hand, the flexible cable can only exert tension and can not push the moving target[5]. Moreover, due to the flexible cable itself is flexible, its dynamic trajectory will deviate from its equilibrium position [6]. Under the effect of the outside world, due to the stiffness of flexible drive is smaller, even long rope change very slowly, the moving target vibration also can appear around its equilibrium position[7]. Therefore, it is very important to analyze the kinematics and dynamics of the flexible cable drive simulation system[8].

Regarding the above preamble, the kinematics and dynamics analysis of the flexible cable drive simulation system is carried out. The relationship between the position of moving target and the speed and acceleration of flexible cable and the length of flexible cable is obtained, and the force condition of the flexible cable is obtained when the moving target is in any position.

\section{Kinematics and Dynamics Modeling of the Under Study Flexible Drive Simulation System}

In this section, kinematics and dynamics analysis are carried out for the plane flexible drive target movement simulation system. Fig. 1 shown the model of the plane flexible drives the target motion simulation system. The four flexible drive units drive the release of the cable, and control movement targets in the plane $\mathrm{X}$ direction and $\mathrm{Y}$ direction. The main components of the flexible drive simulation system include the driving part, the measuring part and the control part. The four driving units are fixed in the four corners of the experimental apparatus. Each driver unit includes the servo motor, reel, 
tension sensor and encoder. The servo motor in the drive unit is connected to the coiling block, and the coder is installed on the servo motor to record the rotation number of the servo motor. The cable is coiled evenly around the coiling block, and the coiling block can be driven by the control of the motor. The cable from the coiling block through the fixed pulley block fixed to the four corners of the moving plane. The cable connected with the pulling force sensor on the moving target, and then connected with the moving target. The tension sensor can detect the tension of the flexible cable in real time. One end of each flexible cable is connected with a moving target, and the other end is connected with a drive unit. The flexible cable drive simulation system realizes the control of the length of the flexible cable by the rotation of the servo motor, thus, the moving target is controlled and the moving task is completed. In Fig.1, the moving target point $\mathrm{P}$ is the origin. $\mathrm{OX}$ for horizontal direction, $\mathrm{OY}$ as the vertical direction. Ignore the size of the fixed pulley, and reduce the fixed pulley to one point, namely the $\mathrm{Ai}(\mathrm{i}=1,2,3,4)$. The size of roller is quite small to the flexible drive simulation system, thus, this simplification does not affect the characteristics of the whole mechanical system.

Initial state of equilibrium under each point coordinates are:

$$
\left\{\begin{array}{c}
A_{1}=\left(x_{1}, y_{1}\right)=\left(\frac{-a}{2}, \frac{b}{2}\right) \quad A_{2}=\left(x_{2}, y_{2}\right)=\left(\frac{a}{2}, \frac{b}{2}\right) \\
A_{3}=\left(x_{3}, y_{3}\right)=\left(\frac{a}{2}, \frac{-b}{2}\right) \quad A_{4}=\left(x_{4}, y_{4}\right)=\left(\frac{-a}{2}, \frac{-b}{2}\right)
\end{array}\right.
$$

Fig. 2 represents the flow chart of the simulation system analysis. Through analyzing the kinematics and dynamics of the flexible drive simulation system, get the position of the moving target, velocity and acceleration and long change relations of four flexible cables.

In the process of dynamics analysis, without considering elastic deformation caused by the flexible, think flexible cable stiffness is infinite, which does not consider flexible cable elastic deformation.

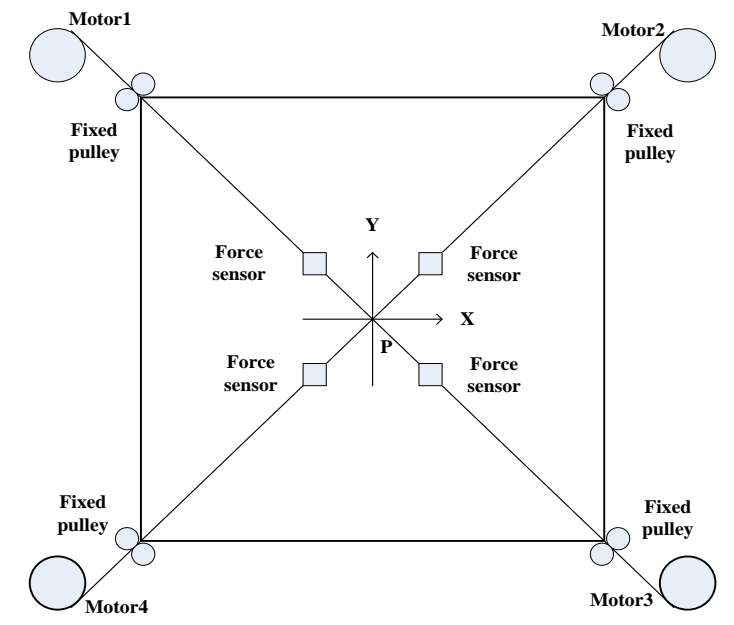

Figure 1. Finite The model of the Planar Flexible Drive Movimg Target

Kinematics Analysis. Inverse kinematics. The movement function is used to calculate the global position and speed of the moving target, In plane coordinate system of the position vector $\mathrm{P}=(\mathrm{x}, \mathrm{y})^{\mathrm{T}}$, roller position vector $A_{k}(k=1,2,3,4)$, the cable length vector $L_{v}$ can be obtained from the:

$$
L^{V}=P-A_{k}
$$

The length of the single cable for:

$$
L_{k}=\left\|L^{V}\right\|=\left\|P-A_{k}\right\|
$$

According to (3), the relationship between the length of the cable and the position of moving target is rewritten as a function: 


$$
L=\Gamma(P)
$$

(4)

The length of the cable, $\mathrm{L}=[\mathrm{L} 1, \mathrm{~L} 2, \mathrm{~L} 3, \mathrm{~L} 4]^{\mathrm{T}}$. To take the derivative (4), the velocity and acceleration of the cable are related to the speed of the motion and acceleration of moving target:

$$
\begin{aligned}
& \dot{L}_{1}=\frac{1}{L_{1}}\left[\dot{x}\left(x+\frac{a}{2}\right)+\dot{y}\left(y-\frac{b}{2}\right)\right] \\
& \ddot{L}_{1}=\frac{1}{L_{1}^{2}}\left[L_{1}\left(\ddot{x}\left(x+\frac{a}{2}\right)+\ddot{y}\left(y-\frac{b}{2}\right)+\dot{x}^{2}+\dot{y}^{2}\right)-L_{1} \dot{L}_{1}^{2}\right]
\end{aligned}
$$

In the same way, we can get the relationship between the speed and acceleration of each of the cable and the speed and acceleration of moving target.

Forward kinematics. The positive solution is that the length of the flexible cable is known to obtain the position of the moving target, that is the mapping from the drive space to the workspace. It is very difficult to solve the forward kinematics, and there are many solutions.

In this paper, based on the parallel robot Jacobian[9], the Newtonian iteration method of solving the multivariate nonlinear equation is adopted to establish the equation of motion. Because the Jacobian is a singular matrix, the flexible cable drives the moving target only on the plane[10]. The four one drives the flexible cable, so the simulation system is over constrained, $\mathrm{J}(\mathrm{P})$ is irreversible, here we introduce the pseudo-inverse of the Jacobian $\left(\mathrm{J}^{-1}\left(\mathrm{P}_{\mathrm{i}}\right)\right)^{+}$.

$$
d P=\left(J^{-1}(P)\right)^{+} d L
$$

Differential substitution differential:

$$
\begin{aligned}
& P^{*}-P^{i} \approx\left(J^{-1}\left(P^{i}\right)\right)^{+}\left(L^{*}-L^{i}\right) \\
& P^{*} \approx P^{i}+\left(J^{-1}\left(P^{i}\right)\right)^{+}\left(L^{*}-L^{i}\right)
\end{aligned}
$$

$\mathrm{P}^{*}$ is the approximate value, and $\mathrm{P}^{*}$ is replaced by $\mathrm{P}_{\mathrm{i}+1}$ :

$$
P^{i+1}=P^{i}+\left(J^{-1}\left(P^{i}\right)\right)^{+}\left(L^{*}-L^{i}\right)
$$

When the $\left(\mathrm{L}^{*}-\mathrm{L}_{\mathrm{i}}\right)$ reaches the accuracy requirement, the iteration is aborted, and if the process is convergent, the $\mathrm{P}_{i+1}$ will converge to $\mathrm{P}^{*}$. For a given convergent accuracy $\varepsilon$, program flowchart is following. Fig. 3 represents the flow chart of the Newton iterative method.

Dynamics Analysis. This section will discuss the performance of the moving target, which is guided by four cables and moves on the plane. The position vector of the moving target is $\mathrm{P}=[\mathrm{x} y]^{\mathrm{T}}$, the long vector of the four cables is $\mathrm{A}_{\mathrm{k}} \mathrm{P}(\mathrm{k}=1,2,3,4), \mathrm{A}_{\mathrm{k}} \mathrm{P}=\mathrm{P}-\mathrm{A}_{\mathrm{k}}$. Therefore, the direction vector of the flexible cable unit is:

$$
u_{k}=\frac{\overline{A_{k} P}}{\left|\overline{A_{k} P}\right|}=\frac{\overline{A_{k} P}}{L_{k}}
$$

The element direction combination matrix of flexible cable is:

$$
J_{d}=\left\{\begin{array}{llll}
u_{1} & u_{2} & u_{3} & u_{4}
\end{array}\right\}
$$

Because the flexible cable is only subjected to tension in the motion. The tension of the flexible cable is $\mathrm{T}=[\mathrm{T} 1, \mathrm{~T} 2, \mathrm{~T} 3, \mathrm{~T} 4]^{\mathrm{T}}$. The mass of moving target is $\mathrm{m}^{\mathrm{p}}$, and the acceleration of moving target is $\mathrm{a}^{\mathrm{p}}$. Then, the dynamic equilibrium equation for moving target is: 


$$
J_{d} \cdot T=m^{p} \times a^{p}
$$

The unit direction matrix $\mathrm{Jd}(\mathrm{P})$ is singular matrix, $\left((\mathrm{Jd}(\mathrm{P}))^{-1}\right)^{+}$is its pseudo-inverse. The equation of mechanical equilibrium is:

$$
T=\left(\left(J_{d}(P)\right)^{-1}\right)^{+} \cdot m^{P} \cdot a^{p}
$$

In this way, the tension of the flexible cables can be obtained at each position of the working space. The system is over constrained, and the tension of flexible cable has multiple solutions. In this paper, the flexible cable is always in a tight state, The direction of the force of four flexible cables is always along flexible cables, thus, the force of the flexible cables can be determined.

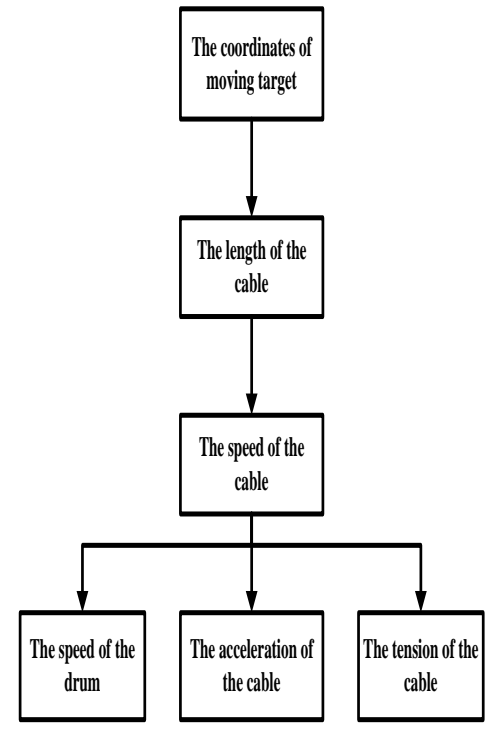

Figure 2. Finite The flow chart of the simulation analysis

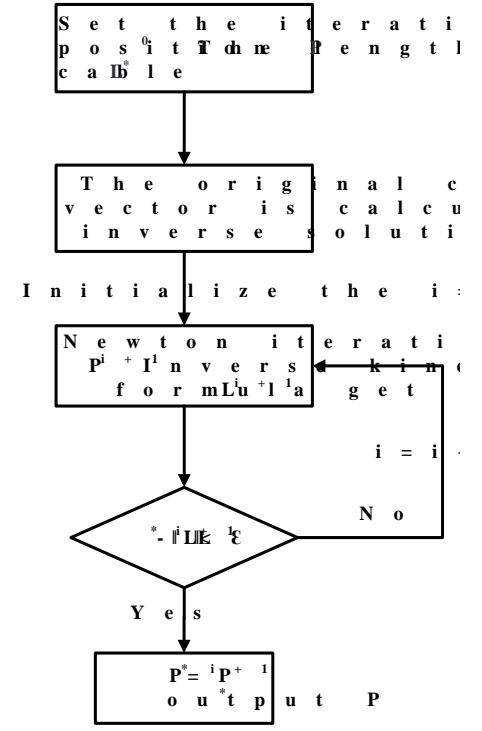

Figure 3. Finite Newton iterative method

\section{A numerical example}

Through the MATLAB software programming, the simulation of flexible cable drive system is simulated.

All geometrical and mechanical parameters of the planar flexible cable drive simulation system are given in Table 1.

Table 1. Parameters of simulation system for planar flexible cable drive.

\begin{tabular}{lll}
\hline Parameter & Symbol & Value \\
\hline Moving target mass & $\mathrm{m}^{\mathrm{P}}$ & $1 \mathrm{~kg}$ \\
Drum radius & $\mathrm{r}$ & $25 \mathrm{~mm}$ \\
$\begin{array}{l}\text { The minimum static tension } \\
\text { The parameters of the planar }\end{array}$ & $\mathrm{F}_{0}$ & $20 \mathrm{~N}$ \\
$\begin{array}{l}\text { flexible driving simulation } \\
\text { system }\end{array}$ & $\mathrm{a} \times \mathrm{b}$ & $1000 \times$ \\
\hline
\end{tabular}

The mass of moving target $\mathrm{m}^{\mathrm{P}}$ is $1 \mathrm{~kg}$. The radius of the drum is $\mathrm{r}=25 \mathrm{~mm}$. The minimum static tension is $F_{0}=20 \mathrm{~N}$. The parameters of the platform for the flexible drive space target is $\mathrm{a} \times \mathrm{b}=1000 \times 1000 \mathrm{~mm}$.

The circular trajectory equation: 


$$
\left\{\begin{array}{l}
x=R \times \cos (\omega \cdot t) \\
y=R \times \sin (\omega \cdot t)
\end{array}\right.
$$

In formula (15), $\mathrm{R}=250 \mathrm{~mm}, \omega=2 \pi / \mathrm{t}$, the period of motion is $10 \mathrm{~s}$. According to the above parameters, the simulation results are as follows:

Fig. 4 shows the trajectory of the moving target. Fig. 5 shows the length of the flexible cables during the movement. Fig. 6 and Fig. 7 show the changes in speed and acceleration of the flexible cables. Fig. 8 shows the force change of the flexible cables.

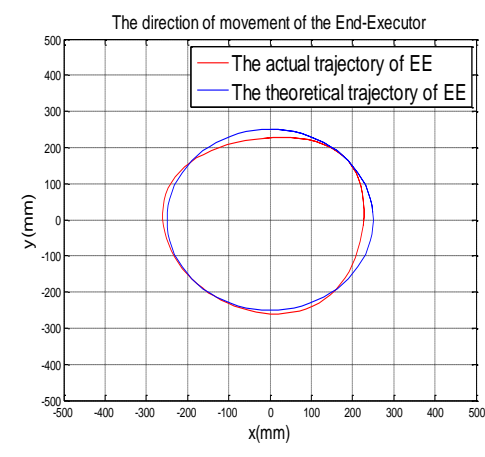

Figure 4. Finite The trajectories of moving

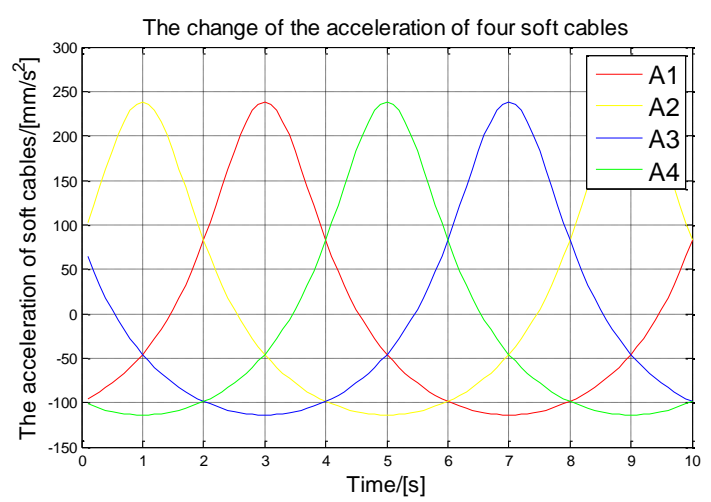

Figure 7. Finite The acceleration change of cables

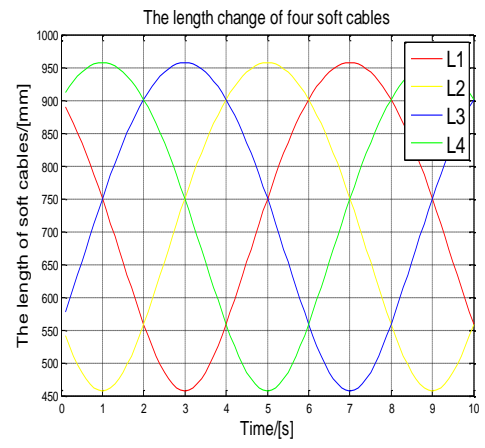

Figure 5. Finite The length change of cables

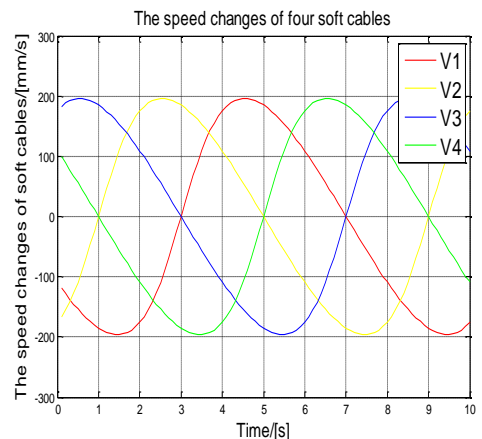

Figure 6. Finite The speed change of cables The tension of the cable

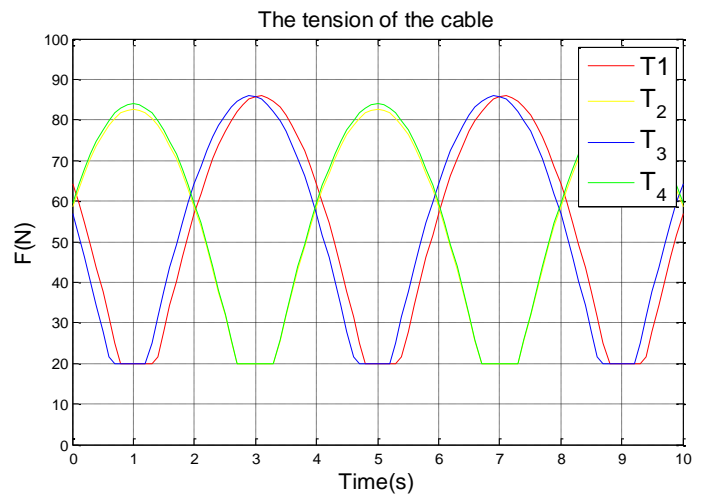

Figure 8. Finite The tension of cables

\section{Conclusions}

According to the simulation results, the positive trajectory of the moving target is almost coincident with the theoretical trajectory, which verifies the feasibility and correctness of the Newton iterative method to solve the kinematics of the flexible cable driven target. When the moving target trajectory is round, the length of the four flexible cables varies symmetrically. In addition, the speed curve and acceleration curve of the four flexible cables are also continuous smooth curve, which is the ideal data for simulating the smooth running of the system. In the course of motion, the force of the four flexible cables is symmetrical. When the moving target runs to the boundary, the tension of the flexible cable reaches its maximum.

In this paper, the kinematics and dynamics of the planar flexible cable drive simulation system are analyzed, which provides a theoretical basis for the next experiment and the control method.

\section{Acknowledgements}

This project is supported by Basic And Advanced Technology Research in Henan Province (No.162300410030) and Innovative Research Team (in Science and Technology) in University of Henan Province (No. 15IRTSTHN008). 


\section{References}

[1] Y.Z. Lei. Recent research progress and prospects of mechanical engineering in china [J]. Bulletin of National Natural Science Foundation of China, 2009, 23(3):134-138. (In Chinese)

[2] B.Zi, Z.Zhu and J.L.Du. Analysis and control of the cable-supporting system including actuator dynamics [J]. Control Engineering Practice. 2011,19(5):491-501. (In Chinese)

[3] A.Girard, J.P.L. Bigué, B.M. O’Brien, T.A. Gisby and I.A. Anderson. Soft two degree of freedom dielectric elastomer position sensor exhibiting linear behavior. IEEE/ASME Transactions on Mechatronics (2015), 20(1), 105-114.

[4] M.X. Liu, D.L. Tan and B.Li. Status and development of reconfigurable modular robots[J]. Robot. 2001,23(3):275-279. (In Chinese)

[5] H. Yuan, E. Courteille and D. Deblaise. Static and dynamic stiffness analyses of cable-driven parallel robots with non-negligible cable mass and elasticity $[\mathrm{J}]$. Mechanism and Machine Theory. 2015,85:64-81.

[6] H. Bayani, M.T. Masouleh and A. Kalhor. An experimental study on the vision-based control and identification of planar cable-driven parallel robots $[\mathrm{J}]$. Roboticcs and Autonomous Systems. 2016,75:187-202.

[7] M.A. Khosravi and H.D. Taghirad. Robust PID control of fully-constrained cable driven parallel robots[J]. Mechatronics, 2014, 24(2):87-97.

[8] G. Liu, Y. Lou and Z. Li. Singularities of parallel manipulators: a geometric treatment[J]. Robotics \& Automation IEEE Transactions on, 2003, 19(4):579-594. (In Chinese)

[9] J.H. Han: Industrial Robot (Huazhong University of Science and Technology Press, China 2015).

[10]B. Zi: Mechanics Analysis and Tracking Control Technology of Hybrid-Driven Based Cable Parallel Robots (Science Press, China 2013). 